\title{
Indivisibility of special values of Dedekind zeta functions of real quadratic fields
}

\author{
by \\ Dongho Byeon (Seoul)
}

1. Introduction and statement of results. For a number field $k$ and a prime number $p$, we denote by $h(k)$ the class number of $k$ and by $\lambda_{p}(k), \mu_{p}(k)$ the Iwasawa $\lambda$-, $\mu$-invariants of the cyclotomic $\mathbb{Z}_{p}$-extension of $k$, where $\mathbb{Z}_{p}$ is the ring of $p$-adic integers.

Let $p$ be an odd prime number. Hartung [3] proved, using the Kronecker class number relation for quadratic forms, that there exist infinitely many imaginary quadratic fields $k$ whose class numbers are not divisible by $p$.

Later, using the idea of Hartung and Eichler's trace formula combined with the $p$-adic Galois representation attached to the Jacobian varieties of certain modular curves, Horie [4] proved that there exist infinitely many imaginary quadratic fields $k$ such that $p$ does not split in $k$ and $p$ does not divide $h(k)$. Thus from a theorem of Iwasawa [7], there exist infinitely many imaginary quadratic fields $k$ with $\lambda_{p}(k)=\mu_{p}(k)=0$.

Let $F$ be a totally real number field. For a prime number $p$, we denote by $n(p)$ the maximum value of $n$ such that the primitive $p^{n}$ th roots $\zeta_{p^{n}}$ of unity are at most of degree 2 over $F$. If $F$ is fixed, we have $n(p)=0$ for all but finitely many $p$. Thus we can put $\omega_{F}=2^{n(2)+1} \prod_{p \neq 2} p^{n(p)}$. Let $\zeta_{F}(s)$ be the Dedekind zeta function of $F$. Serre [11] proved that $\omega_{F} \zeta_{F}(-1)$ is a rational integer. Let $K$ be a totally imaginary quadratic extension over $F$. Define

$$
\lambda_{p}^{-}(K):=\lambda_{p}(K)-\lambda_{p}(F), \quad \mu_{p}^{-}(K):=\mu_{p}(K)-\mu_{p}(F) .
$$

Using a result of Shimizu about the trace formula of Hecke operators and a result of Ohta about the $p$-adic representation of the absolute Galois group over $F$ related to automorphic forms, Naito [8], [9] generalized the above results of Hartung and Horie to the case of totally imaginary quadratic extensions over a totally real number field and obtained the following theorem.

2000 Mathematics Subject Classification: 11R16, 11R23, 11R29.

This work was supported by a new faculty grant from the Seoul National University in 2002 and KOSEF Research Fund (01-0701-01-5-2). 
TheOREm (Naito). Let $F$ be a totally real number field. Let $p$ be an odd prime number which does not divide $\omega_{F} \zeta_{F}(-1)$. Then there exist infinitely many totally imaginary quadratic extensions $K$ over $F$ such that the relative class number of $K$ is not divisible by $p$ and no prime ideal of $F$ over $p$ splits in $K$, that is, $\lambda_{p}^{-}(K)=\mu_{p}^{-}(K)=0$.

Thus it would be interesting to know when or how often $p$ does not divide $\omega_{F} \zeta_{F}(-1)$. In this direction, in this note we will show the following theorem.

TheOREM 1. Let $p$ be an odd prime number. Then there exist infinitely many positive fundamental discriminants $D>0$ such that $p$ does not divide $\omega_{\mathbb{Q}(\sqrt{D})} \zeta_{\mathbb{Q}(\sqrt{D})}(-1)$.

Then, from the above theorem of Naito, we immediately have the following theorem.

TheOREM 2. Let $p$ be an odd prime number. Then there exist infinitely many positive fundamental discriminants $D>0$ such that the real quadratic field $\mathbb{Q}(\sqrt{D})$ has infinitely many totally imaginary quadratic extensions $K$ such that $\lambda_{p}^{-}(K)=\mu_{p}^{-}(K)=0$.

2. Proof of Theorem 1. Let $D$ be the fundamental discriminant of a quadratic number field and $\chi_{D}:=\left(\frac{D}{.}\right)$ the usual Kronecker character. Let $M_{k}\left(\Gamma_{0}(N), \chi\right)$ denote the space of modular forms of weight $k$ on $\Gamma_{0}(N)$ with character $\chi$. Let $r$ and $N$ be nonnegative integers with $r \geq 2$. If $N \not \equiv 0,1$ $(\bmod 4)$, then let $H(r, N)=0$. If $N=0$, then let $H(r, 0):=\zeta(1-2 r)$. If $D n^{2}=(-1)^{r} N$, then

$$
H(r, N):=L\left(1-r, \chi_{D}\right) \sum_{d \mid n} \mu(d) \chi_{D}(d) d^{r-1} \sigma_{2 r-1}(n / d),
$$

where $\sigma_{\nu}(n):=\sum_{d \mid n} d^{\nu}$. Cohen [1] proved the following proposition.

Proposition (Cohen). Let $D \equiv 0$ or $1(\bmod 4)$ be an integer such that $(-1)^{r-1} D=|D|$. Then for $r \geq 2$,

$$
\sum_{N \geq 0}\left(\sum_{\substack{|s| \leq \sqrt{4 N} \\ s^{2} \equiv 4 N(\bmod D)}} H\left(r, \frac{4 N-s^{2}}{|D|}\right)\right) q^{N} \in M_{r+1}\left(\Gamma_{0}(D), \chi_{D}\right),
$$

where $q:=e^{2 \pi i z}$.

Applying this proposition to the case $r=2$, Cohen also obtained the following Kronecker-Hurwitz type formula for $H(2, N)$ :

$$
-30 \sum_{|s| \leq \sqrt{N}} H\left(2, N-s^{2}\right)=\sum_{d \mid N}\left(d^{2}+(N / d)^{2}\right)\left(\frac{-4}{d}\right) .
$$


Lemma. Let $D>0$ be a positive fundamental discriminant. Then

$$
\omega_{\mathbb{Q}(\sqrt{D})}= \begin{cases}2^{3} \cdot 3 & \text { if } D \neq 5 \\ 2^{3} \cdot 3 \cdot 5 & \text { if } D=5 .\end{cases}
$$

For an odd prime number $p \neq 3$, we can choose $l$ to satisfy the following:

(i) $l$ is an odd prime number,

(ii) $l \equiv 3(\bmod 4)$,

(iii) $l^{2} \not \equiv 1(\bmod p)$,

(iv) $\left(\frac{l}{q}\right)=-1$ for all odd prime numbers $q$ with $3 \leq q \leq X$, where $X>5$ is an arbitrarily large number.

Then from (1) and (i), (ii), we have

$$
\sum_{|s| \leq \sqrt{4 l}}\left(-2 H\left(2,4 l-s^{2}\right)\right)=l^{2}-1
$$

From (ii), (iv), for $|s| \leq \sqrt{4 l}$, we have

$$
4 l-s^{2}=D_{l, s} n^{2}
$$

where $D_{l, s}>X>5$ is a positive fundamental discriminant.

From the above lemma, for $|s| \leq \sqrt{4 l}$, we have

$$
\begin{aligned}
-2 H\left(2,4 l-s^{2}\right) & =\omega_{\mathbb{Q}\left(\sqrt{D_{l, s}}\right.} \zeta_{\mathbb{Q}}(-1) H\left(2,4 l-s^{2}\right) \\
& =\omega_{\mathbb{Q}\left(\sqrt{D_{l, s}}\right.} \zeta_{\mathbb{Q}}(-1) L\left(-1, \chi_{D_{l, s}}\right) \sum_{d \mid n} \mu(d) \chi_{D_{l, s}}(d) d \sigma_{3}(n / d) \\
& =\omega_{\mathbb{Q}\left(\sqrt{D_{l, s}}\right.} \zeta_{\mathbb{Q}\left(\sqrt{D_{l, s}}\right)}(-1) \sum_{d \mid n} \mu(d) \chi_{D_{l, s}}(d) d \sigma_{3}(n / d) \in \mathbb{Z} .
\end{aligned}
$$

Finally from (iii), we see that there exist $s$ such that $|s| \leq \sqrt{4 l}$ and $-2 H\left(2,4 l-s^{2}\right) \not \equiv 0(\bmod p), \quad$ i.e., $\quad \omega_{\mathbb{Q}\left(\sqrt{D_{l, s}}\right)} \zeta_{\mathbb{Q}\left(\sqrt{D_{l, s}}\right)}(-1) \not \equiv 0(\bmod p)$.

Since $D_{l, s}>X$ and $X$ is arbitrarily large, for an odd prime number $p \neq 3$, there exist infinitely many positive fundamental discriminants $D$ satisfying $p \nmid \omega_{\mathbb{Q}(\sqrt{D})} \zeta_{\mathbb{Q}(\sqrt{D})}(-1)$.

For the case of $p=3$, we cannot choose $l$ satisfying the above (iii). However we can choose $u, v$ to satisfy the following:

(i) $u, v$ are odd prime numbers,

(ii) $u \equiv 1(\bmod 4)$ and $v \equiv 3(\bmod 4)$,

(iii) $u^{2} v^{2} \not \equiv-1(\bmod 3)$,

(iv) $\left(\frac{u v}{q}\right)=-1$ for all odd prime numbers $q$ with $3 \leq q \leq X$, where $X>5$ is an arbitrarily large number. 
Then by the same method we can easily show that there exist $s$ such that $|s| \leq \sqrt{4 u v}$ and $-2 H\left(2,4 u v-s^{2}\right) \not \equiv 0(\bmod 3)$ and there exist infinitely many positive fundamental discriminants $D$ satisfying $3 \nmid \omega_{\mathbb{Q}(\sqrt{D})} \zeta_{\mathbb{Q}(\sqrt{D})}(-1)$.

3. Remarks. For the case $p=3$ or 5 , by a different method, we can obtain stronger results. From the construction of the Kubota-Leopoldt $p$ adic $L$-function $L_{p}\left(s, \chi_{D}\right)$, the Kummer congruence and the $p$-adic class number formula, we have the following two congruence relations for $\omega_{\mathbb{Q}(\sqrt{D})} \zeta_{\mathbb{Q}(\sqrt{D})}(-1)$, when $D \neq 5$ :

$$
\begin{aligned}
\omega_{\mathbb{Q}(\sqrt{D})} \zeta_{\mathbb{Q}(\sqrt{D})}(-1) & =-2 L\left(-1, \chi_{D}\right) \\
& \equiv-2 L_{3}\left(-1, \chi_{D}\right)(\bmod 3) \\
& \equiv-2 L_{3}\left(1, \chi_{D}\right)(\bmod 3) \\
& \equiv-\frac{4 h(\mathbb{Q}(\sqrt{D})) R_{3}(\mathbb{Q}(\sqrt{D}))}{\sqrt{D}}\left(1-\frac{\chi_{D}(3)}{3}\right)(\bmod 3), \\
\omega_{\mathbb{Q}(\sqrt{D})} \zeta_{\mathbb{Q}(\sqrt{D})}(-1) & =-2 L\left(-1, \chi_{D}\right) \\
& \equiv-2 L_{5}\left(-1, \chi_{5 D}\right)(\bmod 5) \\
& \equiv-2 L_{5}\left(1, \chi_{5 D}\right)(\bmod 5) \\
& \equiv-\frac{4 h(\mathbb{Q}(\sqrt{5 D})) R_{5}(\mathbb{Q}(\sqrt{5 D}))}{\sqrt{5 D}}(\bmod 5) .
\end{aligned}
$$

Thus from (2) and a theorem of Davenport and Heilbronn [2], as refined by Horie and Nakagawa [6], we know that a positive proportion of positive fundamental discriminants $D>0$ satisfy $3 \nmid \omega_{\mathbb{Q}(\sqrt{D})} \zeta_{\mathbb{Q}(\sqrt{D})}(-1)$ and from (3) and a result of Ono [10], we have

$$
\sharp\left\{0<D<X \mid 5 \nmid \omega_{\mathbb{Q}(\sqrt{D})} \zeta_{\mathbb{Q}(\sqrt{D})}(-1)\right\} \gg \sqrt{X} / \log X .
$$

Finally, we mention that Horie and Kimura [5] recently showed that there always exist infinitely many totally imaginary quadratic extensions $K$ over a totally real number field $F$ such that $\lambda_{3}^{-}(K)=\mu_{3}^{-}(K)=0$ whether $\omega_{F} \zeta_{F}(-1)$ is divisible by 3 or not.

\section{References}

[1] H. Cohen, Sums involving the values at negative integers of $L$-functions of quadratic characters, Math. Ann. 217 (1975), 271-285.

[2] H. Davenport and H. Heilbronn, On the density of discriminants of cubic fields II, Proc. Roy. Soc. London Ser. A 322 (1971), 405-420.

[3] P. Hartung, Proof of the existence of infinitely many imaginary quadratic fields whose class number is not divisible by 3, J. Number Theory 6 (1974), 276-278.

[4] K. Horie, A note on basic Iwasawa $\lambda$-invariants of imaginary quadratic fields, Invent. Math. 88 (1987), 31-38. 
[5] K. Horie and I. Kimura, On quadratic extensions of number fields and Iwasawa invariants for basic $\mathbb{Z}_{3}$-extensions, J. Math. Soc. Japan 51 (1999), 387-402.

[6] K. Horie and J. Nakagawa, Elliptic curves with no rational points, Proc. Amer. Math. Soc. 104 (1988), 20-24.

[7] K. Iwasawa, A note on class numbers of algebraic number fields, Abh. Math. Sem. Univ. Hamburg 20 (1956), 257-258.

[8] H. Naito, Indivisibility of class numbers of totally imaginary quadratic extensions and their Iwasawa invariants, J. Math. Soc. Japan 43 (1991), 185-194.

[9] —, Erratum to "Indivisibility of class numbers of totally imaginary quadratic extensions and their Iwasawa invariants", ibid. 46 (1994), 725-726.

[10] K. Ono, Indivisibility of class numbers of real quadratic fields, Compositio Math. 119 (1999), 1-11.

[11] J. P. Serre, Cohomologie des groupes discrets, in: Prospects in Mathematics, Ann. of Math. Stud. 70, Princeton Univ. Press, Princeton, NJ, 1971, 77-170.

School of Mathematical Sciences

Seoul National University

Seoul 151-747, South Korea

E-mail: dhbyeon@math.snu.ac.kr

Received on 5.2.2002

and in revised form on 14.10.2002 